\title{
Burnout em profissionais de Serviço Social durante a pandemia de COVID
}

\section{Burnout in social work professionals during COVID pandemic}

\author{
José Mendes $(1,2)$ \\ Hélia Cabral (3) \\ Flávia Melo (3) \\ (1) INTELECTO - Psicologia \& Investigação, Ponta Delgada, Portugal \\ (2) Inst de Psicologia Cognitiva, Desenvolvimento Humano e Social, Coimbra, Portugal \\ (3) Univ dos Açores, Ponta Delgada, Portugal
}

Recebido: 10/03/2021; Revisto: 29/03/2021; Aceite: 22/04/2021.

https://doi.org/10.31211/rpics.2021.7.1.204

Check for updates

\begin{abstract}
Resumo
Objetivo: O objetivo do estudo visa avaliar a prevalência de burnout em assistentes sociais a exercer funções em Portugal durante a pandemia do SARS-CoV-2. Métodos: Estudo descritivo, quantitativo e transversal realizado entre o mês de novembro de 2020 e março de 2021, onde 416 assistentes sociais, distribuídos por 20 concelhos de Portugal Continental e llhas, responderam a um breve questionário sociodemográfico e ao Inventário de Burnout de Maslach. Resultados: A maioria dos assistentes sociais que participaram no estudo eram mulheres (96,3\%). Verificou-se que $54,7 \%$ desempenhava a sua atividade em serviço social há mais de 11 anos, e 43,8\% eram gestores/responsáveis por mais de 60 processos. No geral, não se verificou burnout nos participantes. Apesar de 82,3\% dos participantes apresentar resultados que indicaram Realização Pessoal, é visível que alguns participantes também apresentaram Exaustão Emocional (45,1\%). Os participantes que executaram horas extra e os participantes com maior número de processos, apresentaram níveis mais altos de Exaustão emocional e de Despersonalização. Os participantes com menos anos de atividade em serviço social foram os que apresentaram níveis mais altos na dimensão de Realização Pessoal. Conclusões: Os participantes do estudo não evidenciam burnout. No entanto, apesar de grande maioria apresentar revelarem níveis mais altos de Realização Pessoal, uma percentagem considerável revela também níveis mais altos de Exaustão Emocional. Estudos futuros devem avaliar padrões biológicos, tais como níveis de cortisol.
\end{abstract}

Palavras-Chave: Exaustão emocional; Despersonalização; Realização pessoal; Burnout; Assistente social.

\section{DI\&D | ISMT}

rpics@ismt.pt https://rpics.ismt.pt
Publicação em Acesso Aberto

(c)2021. O(s) Autor(es). Este é um artigo de acesso aberto distribuído sob a Licença Creative Commons Attribution, que permite uso, distribuição e reprodução sem restrições em qualquer meio, desde que o trabalho original seja devidamente citado.
José Mendes

INTELECTO, Psicologia e desenvolvimento 


\begin{abstract}
Objective: The study aims to assess the prevalence of burnout in social workers working in Portugal during the SARS-CoV-2 pandemic. Method: Descriptive, quantitative, and cross-sectional study carried out between November 2020 and March 2021, 416 social workers from 20 municipalities in mainland Portugal and the islands participated, answering a brief sociodemographic questionnaire and the Maslach Burnout Inventory - Human Services Survey. Results: Most social workers who participated in the study were represented by women (96.3\%). It was found that $54.7 \%$ have been working in social work for more than 11 years, and $43.8 \%$ were managers / responsible for more than 60 processes. In general, there was no burnout in the participants. Although $82.3 \%$ of participants presented results of Personal Accomplishment, it is evident that some participants also presented Emotional Exhaustion (45.1\%). Participants who worked overtime and the participants with a greater number of processes presented higher levels of Emotional Exhaustion and Depersonalization. Participants with fewer years of activity in social work were the ones who presented higher levels in the Personal Accomplishment dimension. Conclusions: Participants in this study do not evidence the presence of burnout. However, although the vast majority present results that reveal personal accomplishment, a considerable percentage reveals higher levels of Emotional Exhaustion. Future studies should assess biological patterns, such as cortisol levels.
\end{abstract}

Keywords: Emotional exhaustion; Depersonalization; Personal accomplishment; Burnout; Social worker.

\title{
Introdução
}

O ano 2020 foi marcado por uma série de mudanças e restrições laborais devido ao elevado número de casos gerados pelo novo coronavírus (SARS-CoV-2, que origina a doença COVID-19). O relatório de situação sobre o COVID-19, emitido no dia 27 de março de 2020 pela Direção Geral de Saúde (DGS), anunciava a suspeita de mais de 25 mil casos, confirmando a existência de 4268 casos positivos (DGS, 2020). Em 27 de março de 2021 , o relatório de situação sobre o COVID-19 apontava já para mais de 820 mil casos de COVID-19 confirmados (DGS, 2021), registando-se em doze meses um aumento extremamente significativo de casos COVID-19 em Portugal. No sentido de conter a pandemia, os decisores políticos e entidades de saúde apresentaram medidas de prevenção e restrição (e.g., isolamento profilático, medidas de higiene, uso de máscara, limitações na mobilidade, teletrabalho, entre outras), com o objetivo de minimizar a disseminação do SARS-CoV-2.

Mamede et al. (2020) mencionam que quando surge o aparecimento do SARS-CoV-2, Portugal já se confrontava com uma recuperação económica, com níveis de desemprego considerados baixos. Neste processo de recuperação económica, o serviço social teve a necessidade de se repensar com o objetivo de superar a descontinuidade de orientações de políticas sociais que vigoravam até o surgimento da Troika (Carvalho et al., 2017). Para estes autores, o Programa de Emergência Social subjugado a uma estratégia global, surge da necessidade da criação de novos paradigmas na intervenção social.

Apesar de o serviço social estar sujeito à forma como a sociedade e o Estado se organizam, é nos assistentes sociais que recai a visibilidade do serviço social (Carvalho \& Pinto, 2015). Essa visibilidade voltou a recair sobre esta classe profissional nos últimos doze meses, em que foram declarados estados de emergência e estados de calamidade, obrigando ao confinamento social e a uma reorganização laboral nas entidades públicas e privadas. Inerente a este confinamento, estão os impactos nos setores económicos, que levam o Instituto de Segurança Social a dispor do Layoff Simplificado para as entidades empregadoras, assim como a necessidade de reforçar o apoio às respostas sociais. Perante a atual situação, prevê-se um maior esforço dos profissionais de serviço social, uma vez que são considerados indispensáveis à sociedade por desenvolverem e executarem políticas sociais (Bracons, 2020). 
No contexto português, a mudança sociopolítica levantou novas questões para a agenda do serviço social, as quais exigiram práticas de intervenção inovadoras correspondentes aos padrões de atuação empregues a nível europeu (Carvalho \& Pinto, 2015). Estes autores referem ainda que passou a ser requisitado ao assistente social a construção de políticas pró-ativas e promotoras da participação comunitária, assentes nas reais necessidades dos indivíduos. Cardoso et al. (2020) referem que os profissionais de serviço social têm, como funções, assegurar o acesso dos cidadãos aos seus direitos, incluindo a segurança e bem-estar.

A deterioração da economia fez-se sentir em todo o mundo, prevendo-se uma crise profunda devido aos efeitos severos da pandemia no emprego e nos rendimentos (Mamede et al., 2020), tendo todas as organizações laborais sido obrigadas a adaptar a sua atividade profissional. Vaz-Serra (2002) menciona que uma atividade profissional apresenta comumente aspetos positivos (e.g., o que se consegue obter através do trabalho) e aspetos negativos (e.g., não gostar do que se faz, incapacidade de adaptação). No entanto, a imposição de um trabalhador se adequar às mudanças inerentes ao seu quotidiano profissional, pode influenciar o desgaste físico e emocional (Nascimento e Silva, 2019).

A pandemia veio acentuar desigualdades sociais, questionando-se o que seria da população sem um sistema de proteção social (Costa, 2020), no qual a habitação (o espaço doméstico) teve a necessidade de se reinventar, transformando-se às necessidades de cada indivíduo (e.g., sala de aula, local de trabalho) (Drago, 2020). Por outro lado, a probabilidade de os cenários de múltiplas violências (e.g., violência doméstica, familiar, psicológica) se tornarem mais comuns, leva os profissionais de saúde a estarem mais cientes do aumento das taxas de vitimização (Campbell, 2020). Redondo-Sama et al. (2020) afirmam que, durante a crise pandémica, o serviço social enfrentou momentos desafiadores para atender às necessidades urgentes num cenário considerado incerto; em que a COVID-19 além de causar preocupações com a saúde física, ocasiona também um número de problemas psicológicos e mentais, inclusive o stress (Salari et al., 2020).

O stress é um conceito comumente utilizado no nosso quotidiano, mas só se revela prejudicial quando um indivíduo se localiza em um dos extremos, por exemplo, passar de uma rotina dinâmica à mon otonia ou o indivíduo não conseguir gerir as exigências com que se confronta (Vaz-Serra, 2002). Contudo, este autor defende a existência de uma relação entre o trabalho, o indivíduo e o stress, onde a sobrecarga do trabalho urge quando o indivíduo sente uma incapacidade de responder às exigências laborais. Em 1974, Freudenberger percebeu a existência de uma constelação de sintomas em resposta à luta diária dos profissionais de saúde cuidarem dos seus doentes. A observação destes sinais e sintomas (e.g., fadiga, frustração, irritabilidade, ineficácia profissional, entre outros) fez assim emergir o conceito de burnout.

O burnout refere-se a um conjunto de sinais e sintomas psicológicos que surgem como resposta a uma experiência individual de stress interpessoal crónico prolongado que envolve a conceção que o indivíduo tem de si mesmo e dos outros no trabalho (Maslach \& Leiter, 2016a, 2016b). Maslach et al. (2001) defendem que o burnout pode ocorrer devido a determinados fatores situacionais (e.g., características do trabalho e da organização) e fatores individuais (e.g., características demográficas, características da personalidade, atitude perante o emprego), apresentando-se em três dimensões: Exaustão Emocional, Despersonalização e Realização Pessoal. Maslach et al. (1997) defendem que sete opções de resposta possibilitam aos investigadores uma maior certeza sobre o valor de cada escala. De facto, as opções de resposta do MBI-HSS não apresentam um ponto médio, o que na opinião de Chyung et al. (2017) evita que os participantes o possam usar quando se confrontam com a indecisão de escolher uma opção de resposta. Os assistentes sociais, profissionais que desenvolvem uma intervenção por meio do estabelecimento de uma relação profissional com outras pessoas, vêem-se comprometidos a solucionar problemas sociais, emocionais 
e/ou físicos que, na maioria das vezes, despertam, em quem os vivencia, sentimentos prejudiciais ao seu bemestar e qualidade de vida (Maslach \& Jackson, 1981). Na contemporaneidade, a intervenção social é confrontada com "uma classe de novos pobres", que carece, inevitavelmente, da criação e implementação de respostas sociais impulsionadoras da capacitação/empowerment dos indivíduos e da sociedade (Carvalho \& Pinto, 2015). Estes autores defendem que "a profissão do Serviço Social tem de assegurar o seu lugar nos novos contextos sociodemográficos, políticos, econômicos, tecnológicos e culturais" (2015, pp. 82-83), demonstrando a capacidade de intervir rápida e eficazmente com poucos recursos sobre os problemas sociais (SánchezMoreno et al., 2015).

Peinado e Anderson (2020) identificaram o número de casos, os salários, a limitação de recursos, as restrições de tempo e os prazos reduzidos, os dilemas éticos e o tipo de instituição como alguns fatores que podem propiciar burnout em assistentes sociais. Estes autores defendem ainda que o decorrer do tempo e o acumular de funções gera um aumento de stress que coloca em causa o bem-estar e satisfação com o trabalho.

Salari et al. (2020) defendem que a COVID-19 não afeta somente a saúde física, mas também a saúde psicológica, exigindo-se um maior conhecimento sobre o impacto desta doença durante a pandemia. Perante a necessidade das respostas sociais advindas das restrições implementadas no combate à pandemia, os profissionais em Serviço Social despenharam funções primordiais na resposta à emergência social. Assim, e em virtude da escassez de estudos desenvolvidos em Portugal neste âmbito, principalmente num período em que decorre o segundo estado de emergência, revelou-se pertinente a realização deste estudo, com os seguintes objetivos: i) verificar a fiabilidade do instrumento MBI-HSS numa amostra de assistentes sociais portugueses durante a pandemia SARS-CoV-2; ii) analisar a possível existência de burnout; iii) explorar correlações entre as dimensões do MBI-HSS e as variáveis de domínio profissional; e iv) pesquisar a existência de diferenças estatisticamente significativas nas variáveis dependentes entre as categorias de variáveis independentes.

\section{Método}

Estudo descritivo, quantitativo e transversal, representado por uma amostragem de conveniência e acidental.

\section{Participantes}

Foram definidos como critérios de inclusão: i) desempenhar a atividade de assistente social e ii) exercer funções em território português. Participaram no presente estudo 416 assistentes sociais, no entanto, aplicados os critérios de inclusão (desempenhar a atividade profissional e em território português), procedeu-se à eliminação de 10 participantes (dois responderam estar no desemprego e oito a desempenharem funções no estrangeiro), perfazendo a amostra final de 406 assistentes sociais, 391 do sexo feminino (96,3\%) e 15 do sexo masculino (3,7\%), com idades compreendidas entre os 19 e os 63 anos $(M=38,11 ; D P=8,93)$. A maioria dos participantes dispunha de um horário fixo ( $n=385 ; 94,8 \%$ ), tinha uma carga horária de 35 horas semanais ( $n=311 ; 76,6 \%$ ) e executavam horas extra $(n=312 ; 76,8 \%)$. A Tabela 1 , apresenta outras características sociodemográficas e profissionais e a Figura 1, apresenta a distribuição dos participantes pelos concelhos de Portugal. 


\section{Tabela 1}

\section{Caracterização da Amostra}

\begin{tabular}{|c|c|c|}
\hline \multirow{2}{*}{ Características sociodemográficas e profissionais } & \multicolumn{2}{|c|}{ Frequências } \\
\hline & $n$ & $\%$ \\
\hline \multicolumn{3}{|l|}{ Estado Civil } \\
\hline Solteiro (a) & 127 & 31,3 \\
\hline Casado (a) & 173 & 42,6 \\
\hline Divorciado (a) & 30 & 7,4 \\
\hline União de Facto & 71 & 17,5 \\
\hline Outro & 5 & 1,2 \\
\hline \multicolumn{3}{|l|}{ Habilitações Académicas } \\
\hline Licenciatura & 325 & 80 \\
\hline Mestrado & 68 & 16,7 \\
\hline Doutoramento & 5 & 1,2 \\
\hline Pós-Graduação & 5 & 1,2 \\
\hline Outro & 3 & 0,7 \\
\hline \multicolumn{3}{|l|}{ Tempo de exercício profissional em Serviço Social } \\
\hline $1-5$ anos & 121 & 29,8 \\
\hline $6-10$ anos & 63 & 15,5 \\
\hline 11 ou mais anos & 222 & 54,7 \\
\hline \multicolumn{3}{|l|}{ Tipo de instituição na qual exerce funções } \\
\hline IPSS & 196 & 48,4 \\
\hline Instituto Privado & 32 & 7,9 \\
\hline Instituto Público & 163 & 40,1 \\
\hline Não responderam & 15 & 3,6 \\
\hline \multicolumn{3}{|l|}{ Tempo de exercício profissional na mesma instituição } \\
\hline $1-5$ anos & 191 & 47 \\
\hline $6-10$ anos & 64 & 15,8 \\
\hline 11 ou mais anos & 151 & 37,2 \\
\hline \multicolumn{3}{|l|}{ Número de casos de que é gestor/responsável } \\
\hline $1-20$ & 62 & 15,3 \\
\hline $21-40$ & 95 & 23,4 \\
\hline $41-60$ & 71 & 17,5 \\
\hline$>60$ & 178 & 43,8 \\
\hline \multicolumn{3}{|l|}{ Prática de algum tipo de exercício físico } \\
\hline $\operatorname{Sim}$ & 142 & 35 \\
\hline Não & 264 & 65 \\
\hline \multicolumn{3}{|l|}{ Considerou alterar de profissão no último mês } \\
\hline Sim & 147 & 36,2 \\
\hline Não & 259 & 63,8 \\
\hline \multicolumn{3}{|l|}{ Considerou mudar de instituição no último mês } \\
\hline $\operatorname{Sim}$ & 185 & 46,6 \\
\hline Não & 221 & 54,4 \\
\hline \multicolumn{3}{|l|}{ Esteve de baixa nos últimos 6 meses } \\
\hline $\operatorname{Sim}$ & 68 & 16,7 \\
\hline Não & 338 & 83,3 \\
\hline
\end{tabular}

Nota. $N=406$. 


\section{Figura 1}

Distribuição dos Participantes pelos Concelhos de Portugal

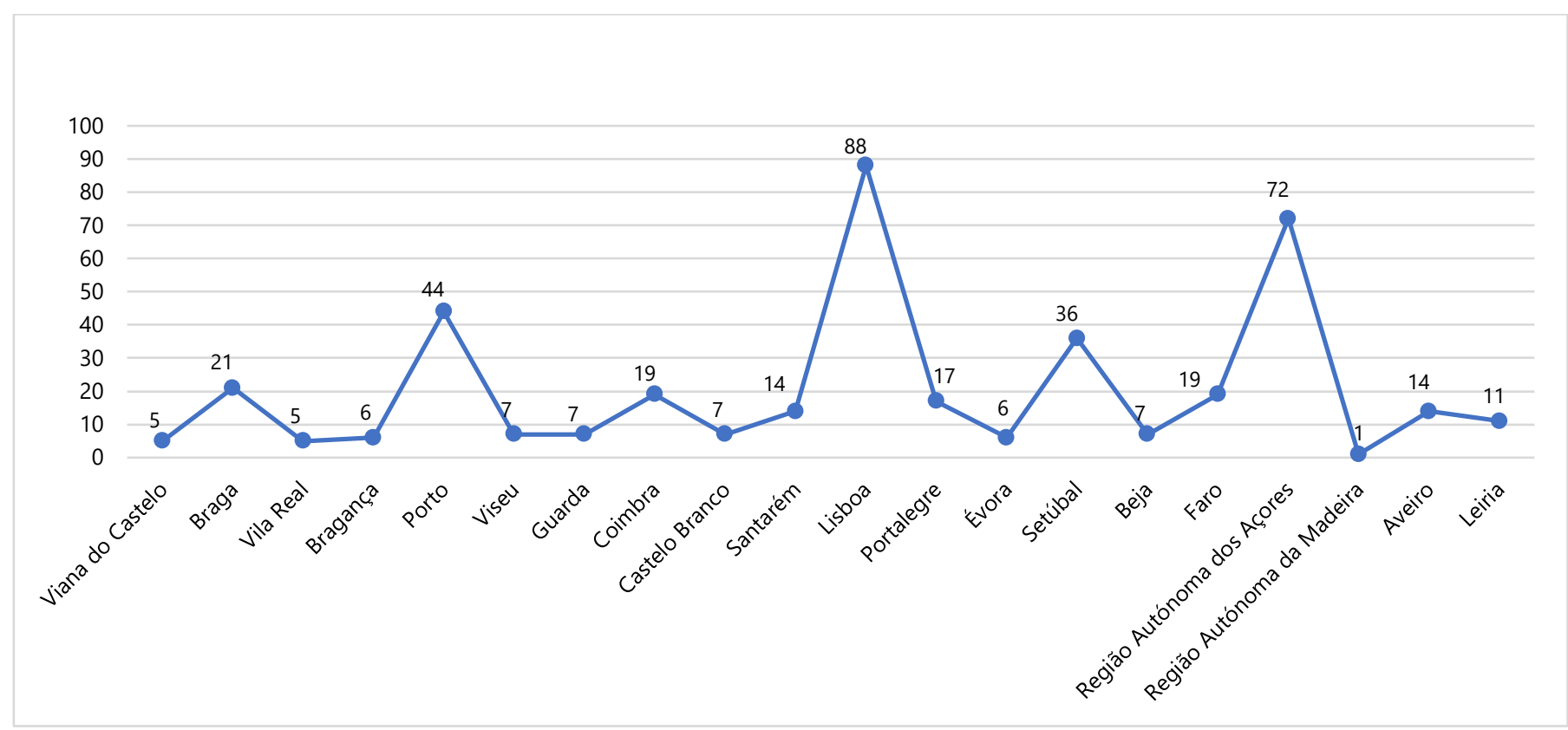

\section{Instrumentos}

\section{Questionário Sociodemográfico}

Elaborou-se um Questionário Sociodemográfico que integrou questões relativas às características pessoais, sociais e profissionais (e.g., sexo; idade; estado civil; habilitações académicas; categoria profissional; tempo de exercício profissional; tipo de instituição; área de intervenção; tempo de exercício profissional na instituição; tipo de horário; carga horária; horas extra; realização de atividades do trabalho em casa; filhos e número de filhos; prática de exercício físico; ponderar alterar de profissão e/ou instituição; e baixa médica).

\section{Inventário de Burnout de Maslach (MBI-HSS)}

O MBI-HSS (Maslach Burnout Inventory - Human Services Survey; versão original de Maslach e Jackson, 1981; tradução e validação para a população portuguesa de Semedo, 2009) avalia o burnout em profissionais que trabalham em serviços humanos e de saúde. O MBI-HSS integra 22 itens com seis opções de resposta $(0=$ nunca, 1 = algumas vezes por ano ou menos, 2 = uma vez por mês, 3 = algumas vezes por mês, $4=$ uma vez por semana, 5 = várias vezes por semana, e 6 = todos os dias) agrupados em três dimensões: i) Exaustão Emocional (9 itens), ii) Despersonalização (5 itens), e iii) Realização Pessoal (8 itens). Considera-se que um indivíduo apresenta burnout quando obtém pontuações elevadas nas dimensões Exaustão Emocional e Despersonalização e baixas pontuações na dimensão Realização Pessoal. A folha de pontuação e interpretação do MBI-HSS apresenta uma categorização (baixo, moderado e elevado) para cada dimensão, no entanto, são desconhecidos estudos que utilizem a categorização originalmente apresentada por Maslach e Jackson (1981). O coeficiente de fiabilidade da versão 
portuguesa apresenta um bom índice de consistência interna para o fator Exaustão Emocional ( $\alpha$ de Cronbach = 0,84) e Realização Pessoal ( $\alpha$ de Cronbach $=0,81$ ), enquanto que o índice de consistência interna para o fator Despersonalização é considerado abaixo do aceitável ( $\alpha$ de Cronbach = 0,57).

\section{Procedimentos}

O presente estudo iniciou-se com a aplicação de dez questionários em papel a assistentes sociais residentes na ilha de São Miguel (Região Autónoma dos Açores), passando posteriormente para a divulgação on-line devido às medidas de restrição. Os primeiros resultados incentivaram a extensão do estudo preliminar a uma amostra maior. Neste sentido, através da plataforma Google Forms foram disponibilizados os dois instrumentos (Questionário Sociodemográfico e MBI-HSS), divulgados nas redes sociais (e.g., Facebook, mais especificamente no grupo Espaço do Assistente Social, com publicação aceite pelo administrador do grupo) e solicitada a participação através do envio do pedido de participação via correio eletrónico. A recolha da amostra decorreu entre o período de novembro de 2020 e março de 2021, tendo sido superado o primeiro confinamento geral e estando a decorrer o segundo confinamento geral. Obedeceram a todos os procedimentos éticos, representados pelo consentimento de informação, participação livre e espontânea, tendo sido assegurada a utilização dos dados recolhidos, única e exclusivamente para efeitos do estudo em causa. Foram garantidos a confidencialidade e anonimato dos participantes.

\section{Análise Estatística}

Para o tratamento e a análise dos dados recolhidos, utilizou-se o software IBM SPSS Statistics, versão 27, tendose analisado estatísticas descritivas e o alfa de Cronbach. Procedeu-se ao cálculo do coeficiente de Spearman (-1 $\leq R_{S} \leq 1$ ) por não se ter verificado o pressuposto de normalidade da variável sob estudo. Marôco (2018) menciona que especialmente em amostras grandes, os testes paramétricos (ANOVA ou $t$-Student) são bastante robustos quando a distribuição em estudo não é normal e quando a distribuição sob estudo apresenta considerável assimetria $(s k<3)$ e achatamento $(k u<8)$. Verificando-se estes pressupostos da normalidade, utilizaram-se $t$ Student e ANOVA para amostras independentes, de forma a verificar se havia diferenças estatísticas entre as variáveis, tendo em consideração o tamanho do efeito (TDE) (Espírito-Santo \& Daniel, 2015, 2018). A análise do teste ANOVA foi robustecida pelo teste de Tukey HSD, para a averiguação das diferenças estatisticamente significativas (Marôco, 2018).

Perante a ausência de uma categorização nas dimensões do MBI-HSS em estudos psicométricos portugueses e a amostra em estudo não seguir uma distribuição normal, optou-se pela criação de um ponto médio $\left(x_{i}^{\prime}=\frac{L i+L s}{2}\right)$ de forma analisar os resultados de cada dimensão, uma vez que a média e o desvio padrão só são bem empregues quando a amostra segue uma distribuição normal (Rodrigues et al., 2017).

\section{Resultados}

Determinou-se a homogeneidade e a fiabilidade do MBI-HSS $\left[K M O=0,92\left(X^{2}{ }_{(231)}=5639,09 ; p<0,001\right)\right.$, tendose obtido coeficientes alfa de Cronbach considerados elevados para a dimensão Exaustão Emocional $(\alpha=0,93)$ e dimensão Realização Pessoal ( $\alpha=0,92)$, apresentando uma baixa fiabilidade para a dimensão Despersonalização $(\alpha=0,70)$ (Marôco \& Garcia-Marques, 2006). Tendo em consideração o ponto médio da 
cotação de cada dimensão, através da análise das médias das dimensões que constituem o MBI-HSS, verificouse que na amostra total dos participantes, os participantes em estudo apresentaram valores que não indicam a presença de burnout; isto é, apresentaram pontuações baixas na dimensão Exaustão Emocional $(M=25,53 ; D P$ $=13,07)$ e Despersonalização $(M=5,81 ; D P=5,45)$, e pontuações elevadas na dimensão Realização Pessoal ( $M$ = 33,20; $D P=10,42)$. Através da Tabela 2, é possível verificar que 45,1\% dos participantes apresentaram um valor superior ao ponto médio na dimensão Exaustão Emocional, 6,4\% na dimensão Despersonalização e 17,7\% dos participantes apresentam um resultado inferior na dimensão Realização Pessoal.

\section{Tabela 2}

Ponto Médio das Dimensões do MBI-HSS e Percentagens dos Resultados Inferiores e Superiores ao Ponto Médio

\begin{tabular}{ccc}
\hline Exaustação Emocional & Despersonalização & Realização Pessoal \\
\hline$x_{i}^{\prime}=27$ & $x_{i}^{\prime}=15$ & $x_{i}^{\prime}=24$ \\
\hline$\leq 27=54,9 \%$ & $\leq 15=92,6 \%$ & $\leq 24=17,7 \%$ \\
$>27=45,1 \%$ & $>15=7,4 \%$ & $>24=82,3 \%$ \\
\hline
\end{tabular}

Nota. $N=406 . \mathrm{MBI}-\mathrm{HSS}=$ Maslach Burnout Inventory - Human Services Survey $; x_{i}^{\prime}=\frac{L i+L S}{2}$.

Ao verificar a intensidade da associação entre as dimensões do MBI-HSS e as variáveis relacionadas com o quotidiano pessoal (e.g., prática de exercício físico) e as variáveis profissionais (e.g., mudar de instituição, mudar de profissão), verificaram-se níveis de intensidade fraca para a maioria das variáveis, com exceção da dimensão Exaustão Emocional que apresentou uma correlação moderada negativa e significativa $\left(R_{S}=-0,39 ; p<0,01\right)$ com a variável Considerar mudar de instituição no último mês.

\section{Tabela 3}

Correlação entre as Dimensões do MBI-HSS e as Variáveis Pessoais e Profissionais

\begin{tabular}{|c|c|c|c|c|c|c|c|c|c|c|}
\hline & 1 & 2 & 3 & 4 & 5 & 6 & 7 & 8 & 9 & 10 \\
\hline 1. Exaustão Emocional & - & $0,48^{* * *}$ & 0,16 & $-0,24 * * *$ & $0,11^{*}$ & $0,13^{*}$ & $-0,40 * * *$ & $-0,49 * * *$ & 0,72 & $-0,08$ \\
\hline 2. Despersonalização & & - & $-0,23 * * *$ & $-0,11^{*}$ & $0,15^{* *}$ & 0,01 & $-0,16^{* *}$ & $-0,28 * * *$ & $-0,22$ & $-0,22 * * *$ \\
\hline 3. Realização Pessoal & & & - & $0,10^{*}$ & $-0,01$ & $-0,12$ & $0,16^{* *}$ & $0,17^{* *}$ & $0,13^{*}$ & $0,12^{*}$ \\
\hline 4. Executa horas extra & & & & - & $-0,03$ & $-0,04$ & $0,17^{* * *}$ & $0,10^{*}$ & $-0,04$ & 0,04 \\
\hline 5. Número de casos de que é responsável & & & & & - & $-0,03$ & $-0,03$ & $-0,05$ & 0,06 & $-0,12 *$ \\
\hline 6. Prática de exercício físico & & & & & & - & $-0,04$ & $-0,04$ & 0,01 & $-0,04$ \\
\hline 7. No último mês considerou mudar de instituição & & & & & & & - & $0,59 * * *$ & 0,04 & 0,09 \\
\hline 8. No último mês pensou em alterar de profissão & & & & & & & & - & 0,07 & 0,04 \\
\hline 9. Nos últimos seis meses esteve de baixa & & & & & & & & & - & $-0,05$ \\
\hline 10. Domínio do campo de intervenção & & & & & & & & & & - \\
\hline
\end{tabular}

Nota. $N=406 . \mathrm{MBI}-\mathrm{HSS}=$ Maslach Burnout Inventory - Human Services Survey.

${ }^{*} p<0,05 ; * *<0,01 ; * * p<0,001$.

$\mathrm{Na}$ realização do teste $t$-Student para a comparação de médias de dois grupos independentes, verificaram-se diferenças estatisticamente significativas nas dimensões Exaustão Emocional $\left[t_{(404)}=4,94 ; p<0,001 ; d\right.$ Cohen $\left.=0,58\right]$, Despersonalização $\left[t_{(404)}=2,36 ; p=0,019 ; d\right.$ Cohen $\left.=0,27\right]$ entre as categorias da variável Executa Horas Extra. 
Verificaram-se também diferenças estatisticamente significativas na Exaustão Emocional entre as categorias da variável Praticar exercício físico $\left[t_{(404)}=-2,54 ; p<0,01 ; d\right.$ Cohen $\left.=0,26\right]$.

Através da Tabela 4, é possível verificar diferenças estatisticamente significativas nas três dimensões do MBI-HSS entre as categorias das variáveis profissionais, apresentando um TDE considerado grande para a dimensão Exaustão Emocional e médio para as dimensões Despersonalização e Realização Pessoal.

\section{Tabela 4}

Dimensões do MBI-HSS Consoante Considerou Mudar ou Não de Instituição/Profissão

\begin{tabular}{|c|c|c|c|c|c|c|c|}
\hline Dimensões MBI-HSS & Variáveis Profissionais & $N$ & $M$ & $D P$ & $t$ & $p$ & $d$ \\
\hline & Considerou Mudar de I & & & & & & \\
\hline \multirow[t]{2}{*}{ Exaustão Emocional } & Sim & 185 & 31,19 & 12,45 & \multirow{2}{*}{8,68} & \multirow{2}{*}{$<0,001$} & \multirow{2}{*}{0,87} \\
\hline & Não & 221 & 20,80 & 11,64 & & & \\
\hline \multirow[t]{2}{*}{ Despersonalização } & Sim & 185 & 6,77 & 5,88 & \multirow{2}{*}{3,30} & \multirow{2}{*}{0,001} & \multirow{2}{*}{0,33} \\
\hline & Não & 221 & 5,00 & 4,95 & & & \\
\hline \multirow[t]{3}{*}{ Realização Pessoal } & Sim & 185 & 31,68 & 10,36 & \multirow{2}{*}{2,72} & \multirow{2}{*}{0,007} & \multirow{2}{*}{0,27} \\
\hline & Não & 221 & 34,48 & 10,33 & & & \\
\hline & Considerou Mudar de & & & & & & \\
\hline \multirow[t]{2}{*}{ Exaustão Emocional } & Sim & 147 & 34,16 & 11,34 & \multirow{2}{*}{11,53} & \multirow{2}{*}{$<0,001$} & \multirow{2}{*}{1,91} \\
\hline & Não & 259 & 20,64 & 11,36 & & & \\
\hline \multirow[t]{2}{*}{ Despersonalização } & Sim & 147 & 7,82 & 6,06 & \multirow{2}{*}{5,81} & \multirow{2}{*}{$<0,001$} & \multirow{2}{*}{0,60} \\
\hline & Não & 259 & 4,67 & 9,57 & & & \\
\hline \multirow[t]{2}{*}{ Realização Pessoal } & Sim & 147 & 31,73 & 9,57 & \multirow{2}{*}{2,16} & \multirow{2}{*}{0,032} & \multirow{2}{*}{0,22} \\
\hline & Não & 259 & 30,04 & 10,80 & & & \\
\hline
\end{tabular}

Nota. $N=406 . \mathrm{MBI}-\mathrm{HSS}=$ Maslach Burnout Inventory - Human Services Survey. TDE considerado insignificante $(<0,19) ;$ pequeno $(0,20-0,49)$, médio $(0,50-0,79)$, grande $(0,80-1,29)$ e muito grande $(>1,30)$ (cit. Tabela 3 em Espírito-Santo \& Daniel, 2015, p. 9).

O teste ANOVA permitiu testar as diferenças estatisticamente significativas nas dimensões Exaustão Emocional $\left[F_{(4,401)}=3,30 ; p=0,011 ; \eta^{2}=0,032\right]$ e Realização Pessoal $\left[F_{(4,401)}=3,38 ; p=0,010 ; \eta^{2}=0,033\right]$ entre as categorias da variável Estado Civil. De acordo com o teste post-hoc HSD de Tukey, as diferenças estatisticamente significativas para a dimensão Exaustão Emocional, ocorreram entre o estado civil União de Facto e os estados civis de Casado (IC95\% =-10,77; -0,79; $p=0,014)$ e Divorciado (IC95\% =-16,19; -0,76; $p=0,023)$, enquanto que para a dimensão Realização Pessoal, essas diferenças ocorreram somente entre o estado civil União de Facto e Casado (IC95\% = $8,45 ;-0,50 ; p=0,019)$.

Ao analisar diferenças para a variável Tempo de exercício profissional em Serviço Social, a dimensão Realização Pessoal foi a dimensão que apresentou diferenças estatisticamente significativas $\left[F_{(4,401)}=3,59 ; p=0,029 ; \eta^{2}=\right.$ $0,02]$. As diferenças estatisticamente significativas verificadas para este fator ocorreram entre os participantes com 1-5 anos e 6-10 anos de tempo de exercício profissional em Serviço Social $(I C 95 \%=0,11 ; 7,68 ; p=0,042)$. Avaliadas possíveis diferenças entre a variável número de casos que os participantes são responsáveis/gestores, verificaram-se diferenças estatísticas significativas para a dimensão Exaustão Emocional $\left[F_{(4,402)}=2,74 ; p=0,043\right.$; $\left.\eta^{2}=0,020\right]$ e dimensão Despersonalização $\left[F_{(4,402)}=2,81 ; p=0,039 ; \eta^{2}=0,021\right]$. Essas diferenças na dimensão Exaustão Emocional verificaram-se nos participantes com 1-20 processos e mais de 60 processos $($ IC95\% = -10,38; $-0,49 ; p=0,025)$, verificando as mesmas diferenças na dimensão Despersonalização $($ IC95\% $=-4,12 ; 0,001 ; p=$ 0,05). 
Por fim, foram encontradas diferenças estatísticas nas dimensões Exaustão Emocional $\left[F_{(12,393)}=2,17 ; p=0,012\right.$;

$\left.\eta^{2}=0,06\right]$ e Despersonalização $\left[F_{(12,393)}=2,95 ; p=0,001 ; \eta^{2}=0,08\right]$ entre as categorias da variável Domínio de intervenção. Foi possível verificar essas diferenças entre os participantes que trabalhavam na área de intervenção com Pessoas Idosas e Emprego e Formação Profissional (IC95\% =1,08; 27,99; $p=0,033$ ) para a dimensão Exaustão Emocional. Na dimensão Despersonalização, verificaram-se diferenças entre os participantes que trabalhavam na área de Ação Social/RSI em comparação com os participantes que trabalhavam na área de Famílias, Crianças e Jovens em risco $(I C 95 \%=0,46 ; 7,46 ; p=0,012)$, área da Saúde $(I C 95 \%=0,26 ; 6,39 ; p=0,020)$, área da Mobilidade Humana (IC95\% =0,17; 9,05; $p=0,034)$, e área da Educação (IC95\% =0,07; 10,82; $p=0,043)$.

No presente estudo não se verificaram diferenças estatisticamente significativas nas dimensões do MBI-HSS entre as categorias das restantes variáveis (e.g., sexo; tipo de turno; realizar trabalho extra em casa; habilitações académicas; ter estado de baixa médica nos últimos 6 meses; tempo de serviço na instituição; tipo de instituição; carga horária semanal).

\section{Discussão}

A consistência interna do MBI-HSS no presente estudo para os 22 itens, comparada com o estudo original de Maslach e Jackson (1981) e a vários estudos referidos na análise fatorial do MBI-HSS realizada por Vicente et al. (2013), apresenta alfas de Cronbach superiores para as três dimensões. A média geral dos resultados indica que os assistentes sociais que participaram no estudo não apresentam níveis de burnout (médias elevadas de Exaustão Emocional e Despersonalização e médias baixas de Realização pessoal). No entanto, é de salientar que o desconhecimento de estudos psicométricos em Portugal que apresentem uma categorização (leve, moderado, elevado) das dimensões propostas na versão original da MBI-HSS, limitam uma análise de níveis baixos, moderados e elevados de burnout nos participantes em estudo.

Perante a emergência de os assistentes sociais intervirem num contexto incerto (e.g., desconhecido), e o facto de estes já terem implementado práticas que correspondem a padrões europeus (Carvalho \& Pinto, 2015), pode ter contribuído para que estes adquirissem estratégias de coping, habilitando-os a uma maior preparação para atuar. Cardoso et al. (2020) defendem que os assistentes sociais e respetivas equipas demonstram capacidade de adaptação a esta nova conjuntura (COVID-19). Por outro lado, Carvalho (2020) menciona que o uso das tecnologias contribuiu para que estes profissionais desempenhassem as suas funções remotamente, adaptando a sua forma de intervir (e.g., satisfazer necessidades emergentes da pandemia).

Analisadas as correlações entre as dimensões do MBI-HSS e as variáveis pessoais, sociais e profissionais, verificaram-se baixas correlações significativas, com exceção de uma correlação moderada entre a variável Considerar mudar de instituição com a dimensão Exaustão Emocional e a variável Considerar mudar de profissão com a dimensão Despersonalização. A validação original do MBI-HSS demonstrou uma relação entre as variáveis profissionais e o burnout (Maslach \& Jackson, 1981). A pressão no ambiente de trabalho originado pela pandemia SARS-CoV-2, pode ter influenciado a falta de energia e entusiasmo, possivelmente encoberta pela falta de que não seja percetível quer pelos participantes em estudo ou quer pelas próprias instituições que providenciam as condições laborais. Por outro lado, esta pressão e aumento do volume de pedidos de apoio social pode ter aumentado os sentimentos insensibilidade emocional levando os participantes a questionar a sua própria existência e a verificarem se a sua atuação como Assistente Social é real. 
Analisadas as variáveis sociodemográficas dos participantes em estudo, somente se verificaram diferenças estatísticas nas duas dimensões do MBI-HSS entre os tipos de Estado Civil, não se tendo apurado diferenças nas restantes variáveis (e.g., sexo, habilitações académicas, ter estado de baixa médica, tempo de instituição, entre outras). Estes resultados apresentam-se muito diferentes de um estudo realizado em Espanha (antes do atual contexto pandémico) que analisou os fatores sociodemográficos e a síndrome de burnout em assistentes sociais, tendo os resultados revelado que as características sociodemográficas apesar de serem variáveis preditores, têm efeitos muito pequenos (Gómez-García et al., 2020). Estes autores verificaram ainda que a variável Ter estado de baixa médica no ano anterior foi um preditor relevante da Exaustão Emocional, enquanto que a variável Estatuto de emprego em tempo integral foi o indicador mais robusto para a dimensão Despersonalização.

Os resultados demonstram que os participantes que referem executar horas extra, apresentam resultados de maior Exaustão Emocional e Despersonalização. Os participantes que são gestores/responsáveis por mais de 60 processos apresentam resultados de maior Exaustão Emocional e Despersonalização quando comparados com os participantes que detêm entre 1 e 20 processos. Carvalho e Neto (2018) ao analisarem os níveis de fadiga, stress e burnout no serviço social de uma IPSS, verificaram uma associação entre a dimensão Exaustão Emocional e a maior sobrecarga de trabalho. Estes autores defendem ainda que uma maior segurança para o trabalhador, uma melhor remuneração, uma menor carga de trabalho e um maior senso de justiça são alguns dos fatores que podem contribuir para manter uma força de trabalho saudável e assim preservar um atendimento de alta qualidade. Por outro lado, os participantes que praticam exercício físico apresentam resultados inferiores na dimensão Exaustão Emocional, em relação aos participantes que não praticam exercício físico. Vários estudos demonstram que a prática de exercício físico tem efeitos positivos para o bem-estar psicológico dos indivíduos, minimizando os níveis de burnout (Appel-Meulenbroek et al., 2020; Ochentel et al., 2018; Sane et al., 2012), e uma menor adesão à prática de exercício físico pode dever-se ao facto de existirem outros tipos de problemas de saúde (Moz et al., 2019).

Verificou-se também diferenças estatisticamente significativas entre os participantes que consideram e não consideram mudar de instituição ou mudar de profissão nas três dimensões do MBI-HSS. Isto é, os participantes que consideram mudar de instituição ou mudar de profissão, apresentam pontuações maiores nas dimensões Exaustão Emocional e Despersonalização, e pontuações menores na dimensão Realização Pessoal. Estes resultados podem indicar que os participantes em estudo possam manifestar algum tipo de sofrimento psíquico. Recentemente Zaki et al. (2020) analisaram uma amostra de trabalhadores em cuidados de saúde, verificando que as equipas avaliadas demonstravam altos níveis de ansiedade e depressão, perda de motivação e características que se correlacionavam com características pós-traumáticas. Por outro lado, os assistentes sociais carecem de apoio de um órgão (e.g., ordem profissional) que zele pelos seus interesses, lhes proporcione maior segurança e justiça nos locais de trabalho (Farr, 2020).

Os participantes em união de facto apresentam Exaustão Emocional, quando comparados com os divorciados e casados. No entanto, estes participantes parecem sentir-se mais realizados profissionalmente, quando comparados com os casados. Estudos mencionam o estado civil como uma variável moderadora da Exaustão Emocional e Realização Pessoal (Aguayo et al., 2017; Cañadas-De la Fuente et al., 2018; Mousavy \& Nimehchisalem, 2014).

Os resultados apontam para o facto de os participantes neste estudo no início da sua carreira (1-5 anos de serviço social) se sentirem mais realizados pessoalmente quando comparados com os participantes com 6-10 anos e 11 ou mais anos. Um estudo que avaliou o stress e o envolvimento laboral de uma amostra de assistentes sociais em 
Portugal, não verificou diferenças ou correlações entre o tempo de serviço, o stress e o envolvimento no trabalho (Teles et al., 2020). No entanto, Hussein (2018) defende que os assistentes sociais mais novos, tendem a ter uma visão mais positiva sobre os níveis de envolvimento no trabalho. Analisando numa outra perspetiva, os assistentes sociais também se deparam com o problema do desemprego ou emprego precário (Dias \& Duque, 2018), podendo o facto de estes profissionais em início de carreira se sentirem realizados profissionalmente por estarem a desenvolver a sua atividade profissional.

Os participantes que trabalham com pessoas idosas demonstram maior Exaustão Emocional, quando comparados com os que trabalham na área do emprego e formação profissional. Quando analisado os participantes que trabalham nas áreas de Ação Social/RSI, evidenciam-se resultados mais elevados na dimensão Despersonalização, quando comparados com as áreas Famílias, Crianças e Jovens em risco, Saúde, Mobilidade Humana, e Educação. Um estudo realizado por Hussein (2018) identificou que o envolvimento e a experiência no trabalho e o suporte administrativo são considerados possíveis fatores moderadores para o burnout nas áreas de serviço com adultos e crianças. Por outro lado, apesar de no presente estudo não se terem verificado diferenças estatisticamente significativas nos diferentes tipos de instituição, Teles et al. (2020) constataram a perceção de níveis de stress mais elevados quando os assistentes sociais pertenciam ao setor laboral privado (e.g., ONG, IPSS).

\section{Limitações}

A presente investigação apresenta várias limitações, no entanto, salienta-se as que se consideram mais relevantes: i) tendo o estudo sido divulgado nas redes sociais, não é possível garantir que todos os participantes em estudo sejam efetivamente assistentes sociais; ii) não se explorou se os participantes frequentaram ações de formação sobre como lidar com o stress; iii) não se questionou se os participantes em estudo recorreram a algum tipo de apoio psicológico; iv) não se exploraram possíveis estratégias de coping que os participantes utilizaram para lidar com as situações menos positivas; v) somente se avaliou a perceção dos assistentes sociais sobre a existência de burnout (e.g., através de um questionário), sugerindo-se que estudos futuros avaliem os padrões biológicos (e.g., níveis de cortisol e DHEA-S que permitem avaliar os níveis de stress).

\section{Conclusão}

A pandemia do SARS-CoV-2 trouxe desafios a todos os profissionais do serviço de saúde e serviço social, tendo os meios de comunicação social, muitas vezes, alertado para o burnout nestes profissionais. A crise instalada em vários setores económicos e o impacto que essa crise se fez sentir nas famílias (algumas já com graves carências económicas) levou a que todos os profissionais de serviço social no ativo dessem uma resposta às necessidades dos cidadãos. Conforme a revisão de literatura apresentada na introdução, a classe profissional do serviço social já havia sido sujeita a várias alterações político-sociais nos últimos anos. Assim, perante um contexto pandémico em que inúmeras famílias se confrontaram com a emergência social, seria expectável que este grupo de profissionais (entre outros) evidenciassem a síndrome de burnout após se terem confrontado com o primeiro confinamento e a vivenciarem o decorrer do segundo confinamento com medidas de restrição ainda mais duras. No total da amostra, a média dos resultados nas dimensões apontam para a ausência de níveis elevados de Exaustão Emocional e Despersonalização e a presença de elevados níveis na dimensão Realização Pessoal. No entanto, numa análise mais detalhada, foi possível verificar que uma percentagem considerável destes profissionais apresenta resultados de Exaustão Emocional acima da média. Se por um lado, “(...) a adaptação dos serviços e a gestão adequada da informação e dos recursos existentes permitiu manter o acompanhamento e 
apoio social aos cidadãos (...)" (Cardoso et al., 2020, p. i), verificou-se que estes profissionais desempenharam mais horas extra e ficaram responsáveis por um maior número de processos, podendo estas variáveis contribuírem para o aumento dos resultados na dimensão Exaustão Emocional e Despersonalização.

Considera-se que o presente estudo se revela importante para a classe profissional do Serviço Social, na medida em que foi possível avaliar os índices da síndrome de burnout nos profissionais que a constituem. Os assistentes sociais estão diariamente, expostos a um possível burnout dado que intervêm junto de um público-alvo que em contexto pandémico pode apresentar problemáticas, muitas vezes complexas e inerentes à emergência social. Assim, estes profissionais podem com o passar do tempo, ficar cada vez mais vulneráveis ao risco de burnout, comprometendo a eficácia e a qualidade da sua intervenção.

$\mathrm{O}$ assistente social afetado por esta síndrome perde a capacidade de atender à singularidade do sujeito com o qual intervém, dificultando uma melhor compreensão dos seus comportamentos, emoções e sentimentos, uma vez que corre o risco de deixar de se envolver verdadeiramente na relação com o cliente. Lembre-se que o desenvolvimento de uma atitude de distanciamento/desinteresse face ao cliente, acrescido do esgotamento profissional e pessoal, diminui a proatividade do profissional e prejudica inevitavelmente a sua intervenção.

Neste contexto, revela-se fundamental a investigação em serviço social voltada para esta temática, possibilitando questões práticas que sustentam a intervenção social mesmo em tempos de pandemia. A investigação nesta área permite uma melhor monitorização da prática profissional dos assistentes sociais, tendo em vista a constante melhoria da qualidade e eficácia dos serviços prestados, segundo o Código Deontológico dos Assistentes Sociais em Portugal, na promoção do "desenvolvimento e a mudança social, a coesão social, o empowerment e a promoção da Pessoa" (Associação de Profissionais de Serviço Social, 2018, p. 5).

Neste sentido, a partir da investigação é analisado se a classe profissional está condicionada por esta síndrome, contribuindo para uma melhor compreensão sobre o que deve ser alterado na intervenção, a um nível micro, meso e macro. Por outro lado, permite apostar na prevenção e na estimulação do bem-estar pessoal e profissional que consequentemente contribui para melhorar a qualidade dos serviços prestados.

Os resultados apresentados revelam-se pertinentes porque apesar de estes intervirem num contexto pandémico que exige profundas alterações na forma de intervir, a maioria destes profissionais apresenta elevada Realização Pessoal, seguido de Exaustão Emocional e por fim a Despersonalização, o que indica que os profissionais em estudo não estão, na sua maioria, com a síndrome de burnout. Assim, a presente investigação permite considerar a possibilidade de que estes profissionais, perante a ausência de uma Ordem Profissional que os represente, estão resilientes às adversidades da profissão de Serviço Social. De forma geral apresentam-se aptos e preparados para lidar com situações de extremo desgaste emocional.

Tais aptidões são também fruto das orientações de entidades públicas e privadas que orientam estes profissionais para a ação. Um destes exemplos é o gabinete de planeamento e estratégia do Instituto de Segurança Social, que disponibilizou em fevereiro de 2020, o "Plano de ação do ISS, IP", apresentando orientações estratégias e medidas que visem o bem-estar do próprio sistema de Segurança Social. No entanto, salienta-se que é necessário ter em atenção que uma significativa percentagem dos assistentes sociais em estudo apresenta, principalmente, níveis de exaustão emocional, sinais que podem exigir a necessidade de se apostar na monitorização da saúde mental e/ou física desses profissionais e para a crucial revisão a fazer sobre as metodologias de intervenção vigentes, visto que, a longo prazo, esta tendência pode comprometer drasticamente a intervenção.

$\mathrm{O}$ estudo divulgado nas redes sociais (e.g., facebook) permitiu algum feedback sobre o estudo em questão. Além das congratulações recebidas por alguns participantes, outros manifestaram a sua opinião de "tristeza" da 
necessidade de uma pandemia para que estudos analisem a possibilidade de existir burnout nos assistentes sociais. Estes comentários obrigam-nos a refletir sobre o "descuidado" que os decisores políticos e talvez algumas instituições públicas/privadas têm sobre uma área profissional que na sua grande maioria, se confronta com situações de vulnerabilidade e emergência social.

Sugerem-se estudos psicométricos da MBI-HSS que considerem a categorização (baixa, moderada, elevada) das dimensões Exaustão Emocional, Despersonalização e Realização Pessoal.

Agradecimentos | Acknowledgements: Os autores agradecem aos coordenadores do grupo Espaço do Assistente Social na rede social do Facebook pelo extraordinário empenho na divulgação do presente estudo.

Conflito de interesses | Conflict of interest: Nenhum | None.

Fontes de financiamento | Funding sources: Nenhuma | None.

Contributos: FM: Revisão da literatura; Recolha e codificação dos dados; Redação e revisão do manuscrito. HC: Revisão da literatura; Recolha e codificação dos dados; Redação e revisão do manuscrito. JM: Revisão da literatura; Recolha dos dados; Análise estatística; Redação, discussão e revisão do manuscrito.

\section{Referências}

Aguayo, R., Vargas, C., Cañadas, G. R., \& De la Fuente, E. I. (2017). Are socio-demographic factors associated to burnout syndrome in police officers? A correlational meta-analysis. Anales de Psicología, 33(2), 383. https://doi.org/f9z6

Appel-Meulenbroek, R., Voordt, T. van der, Aussems, R., Arentze, T., \& Le Blanc, P. (2020). Impact of activity-based workplaces on burnout and engagement dimensions. Journal of Corporate Real Estate, 22(4), $279-296$. https://doi.org/f9z7

Associação de Profissionais de Serviço Social. (2018). Código deontológico dos assistentes sociais em Portugal. APSS. https://bit.ly/3h6iPAC

Bracons, H. (2020). Perceção dos estudantes finalistas de serviço social face ao contexto de emergência Covid-19. Revista Internacional de Educación para la Justicia Social, (9), 3. https://bit.ly/2PQYX9K

Campbell, A. M. (2020). An increasing risk of family violence during the Covid-19 pandemic: Strengthening community collaborations to save lives. Forensic Science International: Reports, 2, 1-3. https://doi.org/dt54

Cañadas-De la Fuente, G., Ortega, E., Ramirez-Baena, L., De la Fuente-Solana, E., Vargas, C., \& Gómez-Urquiza, J. (2018). Gender, marital status, and children as risk factors for burnout in nurses: A meta-analytic study. International Journal of Environmental Research and Public Health, 15(10), 2102. https://doi.org/gfm3xk

Cardoso, J., Vilar, D., \& Casquilho-Martins, I. (2020). Desafios ao serviço social no contexto da COVID-19. Centro Lusíada de Investigação em Serviço Social e Intervenção Social - CLISSIS. https://bit.ly/3nKwtLg

Carvalho, D. T., \& Neto, H. V. (2018). Fadiga, stresse, burnout e estratégias de coping num serviço social de uma IPSS. International Journal on Working Conditions, 15, 113-129. https://bit.ly/338siiW

Carvalho, M. I. (2020). O serviço social na saúde em Portugal e os desafios da Covid-19. Revista Família, Ciclos de Vida e Saúde no Contexto Social, 8, 1086. https://doi.org/f9z8

Carvalho, M. I., \& Pinto, C. (2015). Desafios do Serviço Social na atualidade em Portugal. Serviço Social \& Sociedade, 121 , 66-94. https://doi.org/f9z9 
Carvalho, M. I., Pinto, C., Costa, J. L., Duarte, C., \& Garcia, A. P. (2017). Repensar o serviço social em tempo de crise económica e financeira em Portugal. Trabajo Social Global-Global Social Work, 7(12), 120-145. https://doi.org/f92b

Chyung, S. Y. Y., Roberts, K., Swanson, I., \& Hankinson, A. (2017). Evidence-based survey design: The Use of a midpoint on the Likert scale. Performance Improvement, 56(10), 15-23. https://doi.org/gfgm4w

Costa, A. F. (2020). Desigualdades sociais e pandemia. Em R. M. Carmo, I. Tavares, \& A. F. Cândido (Eds.), Um olhar sociológico sobre a crise Covid-19 em livro. Observatório das Desigualdades, CIES- ISCTE.

Dias, F., \& Duque, E. (2018). O impacto da crise no serviço social. HOLOS, 8, 138-158. https://doi.org/f92d

Direção Geral de Saúde. (2020). Novo coronavírus COVID-19: Relatório de situação. https://bit.ly/3gZot7S

Direção Geral de Saúde. (2021). COVID-19: Relatório de situação. Situação epidemiológica em Portugal. https://bit.ly/3vCSNsN

Drago, A. (2020). Habitação, pandemia, crise. Em R. M. Carmo, I. Tavares, \& A. F. Cândido (Eds.), Um olhar sociológico sobre a crise Covid-19 em livro. Observatório das Desigualdades, CIES-ISCTE.

Espírito-Santo, H., \& Daniel, F. B. (2015). Calcular e apresentar tamanhos do efeito em trabalhos científicos (1): As limitações do $\mathrm{p}<0,05$ na análise de diferenças de médias de dois grupos. Revista Portuguesa de Investigação Comportamental e Social, 1(1), 3-16. https://doi.org/gg9dgs

Espírito-Santo, H. M. A., \& Daniel, F. (2018). Calcular e apresentar tamanhos do efeito em trabalhos científicos (3): Guia para reportar os tamanhos do efeito para análises de regressão e ANOVAs. Revista Portuguesa de Investigação Comportamental e Social, 4(1), 43-60. https://doi.org/f92f

Farr, P. (2020). Social workers of the world unite! A call to unionize the social work profession. International Social Work, 1, 1-5. https://doi.org/f92g

Freudenberger, H. J. (1974). Staff burn-out. Journal of Social Issues, 30(1), 159-165. https://doi.org/bvn2gn

Gabinete de Planeamento e Estratégia. (2020). Plano de ação do ISS, IP. https://bit.ly/2RmXGay

Gómez-García, R., Alonso-Sangregorio, M., \& Llamazares-Sánchez, M. L. (2020). Burnout in social workers and sociodemographic factors. Journal of Social Work, 20(4), 463-482. https://doi.org/f92h

Hussein, S. (2018). Work engagement, burnout and personal accomplishments among social workers: A comparison between those working in children and adults' services in England. Administration and Policy in Mental Health and Mental Health Services Research, 45(6), 911-923. https://doi.org/gfgsqx

Mamede, R. P., Pereira, M., \& Simões, A. (2020). Portugal: Uma análise rápida do impacto da COVID-19 na economia e no mercado de trabalho. Organização Internacional do Trabalho.

Marôco, J. (2018). Análise estatística com o SPSS Statistics (7ạ ed.). Report Number.

Marôco, J., \& Garcia-Marques, T. (2006). Qual a fiabilidade do alfa de Cronbach? Questões antigas e soluções modernas? Laboratório de Psicologia, 4(1), 65-90. https://bit.ly/3ufNQpP

Maslach, C., \& Jackson, S. E. (1981). The measurement of experienced burnout. Journal of Organizational Behavior, 2(2), 99-113. https://doi.org/cfj

Maslach, C., \& Leiter, M. P. (2016a). Burnout. In G. Fink (Ed.), Stress: Concepts, cognition, emotion, and behavior (Vol. 1, pp. 351-357). Elsevier. https://doi.org/dvj6

Maslach, C., \& Leiter, M. P. (2016b). Understanding the burnout experience: Recent research and its implications for psychiatry. World Psychiatry, 15(2), 103-111. https://doi.org/f8twbg

Maslach, C., Jackson, S. E., \& Leiter, M. P. (1997). Maslach burnout inventory. In C. P. Zalaquett \& R. J. Wood (Eds.), Evaluating stress: A book of resources (pp. 191-218). Scarecrow Education. 
Maslach, C., Schaufeli, W. B., \& Leiter, M. P. (2001). Job Burnout. Annual Review of Psychology, 52(1), $397-422$. https://doi.org/b2w8ff

Mousavy, S., \& Nimehchisalem, V. (2014). Contribution of gender, marital status, and age to English language teachers' burnout. Advances in Language and Literary Studies, 5(6), 39-47. https://doi.org/f92m

Moz, A., Andrade, F., Morais, T., \& Vasconcelos-Raposo, J. (2019). Influência da atividade física nos sintomas depressivos de diabéticos e hipertensos. Psychtech \& Health Journal, 2(2), 27-38. https://doi.org/f92n

Nascimento e Silva, G. (2019). (Re)Conhecendo o estresse no trabalho: Uma visão crítica. Gerais: Revista Interinstitucional de Psicologia, 12(1), 51-61. https://doi.org/f92p

Ochentel, O., Humphrey, C., \& Pfeifer, K. (2018). Efficacy of exercise therapy in persons with burnout. A systematic review and meta-analysis. Journal Sports Science \& Medicine, 17(3), 475-484. https://bit.ly/3xJBcBv

Peinado, M., \& Anderson, K. N. (2020). Reducing social worker burnout during COVID-19. International Social Work, 63(6), 757-760. https://doi.org/f92q

Redondo-Sama, G., Matulic, V., Munté-Pascual, A., \& Vicente, I. (2020). Social work during the COVID-19 crisis: Responding to urgent social needs. Sustainability, 12, 1-16. https://doi.org/f92r

Rodrigues, C. F. de S., Lima, F. J. C. de, \& Barbosa, F. T. (2017). Importância do uso adequado da estatística básica nas pesquisas clínicas. Brazilian Journal of Anesthesiology, 67(6), 619-625. https://doi.org/f92s

Salari, N., Hosseinian-Far, A., Jalali, R., Vaisi-Raygani, A., Rasoulpoor, S., Mohammadi, M., Rasoulpoor, S., \& KhalediPaveh, B. (2020). Prevalence of stress, anxiety, depression among the general population during the COVID-19 pandemic: A systematic review and meta-analysis. Globalization and Health, 16(1), 57. https://doi.org/gg5qtv

Sánchez-Moreno, E., Roldán, I.-N. F., Gallardo-Peralta, L. P., \& Roda, A. B. L. (2015). Burnout, informal social support and psychological distress among social workers. British Journal of Social Work, 45(8), $2368-2386$. https://doi.org/f748qp

Sane, M. A., Devin, H. F., Jafari, R., \& Zohoorian, Z. (2012). Relationship between physical activity and it's components with burnout in academic members of Daregaz Universities. Procedia - Social and Behavioral Sciences, 46, 42914294. https://doi.org/f92t

Semedo, C. S. (2009). Bem-estar psicológico no trabalho e acidentalidade laboral em profissionais de saúde [Tese de Doutoramento, Universidade de Santiago de Compostela]. Repositório da Universidade de Santiago de Compostela. https://bit.ly/2RpOKmx

Teles, H. M. M., Ramalho, N. A., Ribeiro, S. M. P., \& Ramalho, V. S. B. (2020). Níveis de estresse e engagement laboral dos Assistentes Sociais em Portugal. Estudos de Psicologia,24(3), 258-268. https://doi.org/f92v

Vaz-Serra, A. (2002). Stress na vida de todos os dias. Gráfica de Coimbra, Lda.

Vicente, C. S., Oliveira, R. A., \& Marôco, J. (2013). Análise fatorial do inventário de burnout de Maslach (MBI-HSS) em profissionais portugueses. Psicologia, Saúde \& Doenças, 14(1), 152-167. https://bit.ly/33aoiOG

Zaki N. F. N., Sidiq. M, Qasim. M, Aranas. B, Hakamy. A, Ruwais. N. A, Alanezi. H, Al Saudi, D. A., Alshahrani, R. B. R, AlThomali, A. B. A, Manzar, M. D., BaHammam A. S., Al-Kaabba, A. F., \& Pandi-Perumal, S. R. (2020). Stress and psychological consequences of COVID-19 on health-care workers. Journal of Nature and Science of Medicine, 3, 299307. https://bit.ly/3y6hYWX 